\title{
Heavy quarkonium dissociation in the finite space of heavy-ion collisions
}

\author{
Jihong Guo, Wu-Sheng Dai, Mi Xie, Yunpeng Liu \\ Department of Physics, Tianjin University, Tianjin 300350, P. R. China
}

\begin{abstract}
The dissociation of heavy quarkonia in the constrained space is calculated at leading order compared with that in infinitely large medium. To deal with the summation of the discrete spectrum, a modified Euler-Maclaurin formula is developed as our numerical algorithm. We find that with the constraint in space, the dissociation of quarkonia at early time becomes negligible.
\end{abstract}

Keywords: Quark-gluon plasma, Relativistic heavy-ion collisions, Heavy quarkonia PACS: 12.38.Mh, 25.75.-q

\section{Introduction}

The quark-gluon plasma (QGP) is believed to be the state of quark matter at extremely high temperature and/or extremely high density. Such conditions can be found in laboratory only by relativistic heavy-ion collisions. The volume in which the QGP is produced is at the same scale as that of a nucleus, and the QGP can-not be detected directly. Heavy quarkonia are important probes of the QGP produced in heavy-ion collisions, since they suffer suppression in the QGP and almost survive the hadron gas. Fruitful results are obtained in experiments [1 $[6]$ including the nuclear modification factors of quarkonia at different energies, rapidities, and transverse momenta. On the other hand, different models are put up to calculate the suppression. The most early idea is that different excited states of quarkonia melt in the QGP sequentially due to the color screening [7, 8]. A different point of view [9] attributes all the observed heavy quarkonia to the thermal balance between the open and hidden heavy flavors. Meanwhile, the calculation based on scattering cross section was put up 10-13], and the regeneration of quarkonia from heavy quarks in the QGP is considered [12, 14, 15]. The properties of heavy quarkonia have also been studied in the framework of effective field theory [16] and lattice QCD [17]. The theories are still under development recent years [18 30].

All above theories focus on the properties of quarkonia in infinitely large medium, while the volume of the QGP is finite in experiments, especially at early time after the collision. One direct consequence is that the spectrum of gluons becomes different in

Email address: yunpeng.liu@tju.edu.cn (Yunpeng Liu)

Preprint submitted to Elsevier

December 5, 2018 
the finite space compared with that in the infinite space at the same temperature, and therefore the dissociation rate of heavy quarkonia differs. In this letter we will discuss the corresponding effect. Note that the longitudinal size of the medium is much smaller than that in the transverse directions, we will assume that the medium is infinitely large in the transverse directions. For simplicity, we only consider the initially produced quarkonia at middle rapidity and take leading order cross section of the gluons dissociation process.

In Section 2, we introduce the model to describe the suppression of quarkonia, where a summation in the spectrum of gluons in the finite space is introduced to replace the integral in the infinite space. To deal with the summation, a modified Euler-Maclaurin Formula is developed in Section 3 as our numerical algorithm. Results of the gluon spectrum and the dissociation rate of quarkonia in the finite space compared with that in the infinite space is shown in Section 4 The effective initial time is also discussed in this section. A short conclusion is given in Section 5 . We take the natural units $\hbar=c=k_{\mathrm{B}}=1$. A pair of square brackets [ ] in an equation within this letter is always used as a floor function.

\section{Dissociation of quarkonia}

In high energy nuclear collisions, the distribution function $f_{H}(\boldsymbol{p}, \boldsymbol{x}, t)$ of heavy quarkonia $H$ at $(\boldsymbol{p}, \boldsymbol{x})$ in the phase space at time $t$ is controlled by the equation [31]

$$
\partial f_{H} / \partial t=-\alpha_{H} f_{H}
$$

at middle rapidity in the lab frame, where $\alpha_{H}(\boldsymbol{p}, \boldsymbol{x}, t)$ is the dissociation rate of $H$ in the hot medium. We have neglected both the leakage effect [31] and the mean field effect 32] on heavy quarkonia.

Before discussing the loss term $\alpha_{H}$ in the finite space, we first write it in the infinite space. For simplicity, we only consider the gluon dissociation process $g+H \rightarrow Q+\bar{Q}$ in the QGP phase, and the loss term $\alpha_{H}$ is

$$
\alpha_{H}(\boldsymbol{p}, \boldsymbol{x}, t)=\frac{1}{2 E_{H}} \int \frac{\mathrm{d}^{3} \boldsymbol{k}}{(2 \pi)^{3} 2 E_{g}} W_{g H}^{Q \bar{Q}}(s) f_{g}(\boldsymbol{k}, \boldsymbol{x}, t),
$$

where $E_{H}$ and $E_{g}$ are the energies of the heavy quarkonium $H$ and the gluon, respectively, in the lab frame. The transition probability $W_{g H}^{Q \bar{Q}}(s)=4 \sigma_{g H} \sqrt{\left(p^{\mu} k_{\mu}\right)^{2}-m_{H}^{2} m_{g}^{2}}$ is a function of $s=\left(p_{\mu}+k_{\mu}\right)^{2}$ with $p_{\mu}$ and $k_{\mu}$ being the four-momenta of $H$ and the gluon, respectively. The gluon mass $m_{g}$ is taken zero. The dissociation cross section [33, 34] is

$$
\sigma_{g H}(\omega)=A_{0} \frac{\left(\omega / \epsilon_{H}-1\right)^{3 / 2}}{\left(\omega / \epsilon_{H}\right)^{5}}
$$

with $A_{0}=2^{11} \pi /\left(27 \sqrt{m_{Q}^{3} \epsilon_{H}}\right)$, where $\omega=\left(s-m_{H}^{2}\right) / 2 m_{H}$ is the gluon energy in the rest frame of $H$. The binding energy is replaced by the threshold energy $\epsilon_{H}=\left(4 m_{Q}^{2}-\right.$ $\left.m_{H}^{2}\right) /\left(2 m_{H}\right)$ in our calculation in order to include the recoiling effect [35], where $m_{Q}$ is the mass of a heavy quark. The distribution function of gluons is assumed to be thermal

$$
f_{g}(\boldsymbol{k}, \boldsymbol{x}, t)=\frac{N_{g}}{2},
$$


where $T(\boldsymbol{x}, t)$ and $u^{\mu}(\boldsymbol{x}, t)$ are the local temperature and four-velocity, respectively, and $N_{g}=16$ is the degeneracy of gluons. The dissociation in the hadron phase is neglected.

For simplicity, we describe the fireball by Bjorken's hydrodynamics, which neglects the transverse flow of the medium, and the entropy density is inversely proportional to time. For the spatial distribution, the entropy is assumed to be proportional to the number density of participants $n_{\text {part }}$. We take the equation of state as that of ideal parton gas. Then we have

$$
T(\boldsymbol{x}, t)=T^{*}\left(\frac{t^{*}}{t} \frac{n_{\mathrm{part}}(\boldsymbol{x})}{n_{\mathrm{part}}(\boldsymbol{x}=\mathbf{0})}\right)^{\frac{1}{3}}
$$

where $T^{*}$ is the temperature at $\boldsymbol{x}=\mathbf{0}$ and $t=t^{*}$. The number density of binary collision $n_{\text {part }}$ is calculated by the Glauber model [36] with the Woods-Saxon density profile $\rho(\boldsymbol{r})=\frac{\rho_{0}}{1+e^{\left(r-r_{0}\right) / a}}$. The specific parameters of ${ }^{197} \mathrm{Au}\left(r_{0}=6.38 \mathrm{fm}, a=0.535 \mathrm{fm}\right.$ and $\rho_{0}=0.169 \mathrm{fm}^{-3}$ ) used in the numerical calculations are from Ref. [37].

Now we consider the loss term $\alpha_{H}$ in the finite space. In relativistic heavy-ion collisions, the QGP only exists in a small region in space, especially at early time after the collision when the longitudinal size is small. For simplicity, we assume that the fireball is infinitely large in the transverse directions and the longitudinal size of the fireball is $L=2 t$ at time $t$ after the collision. The eigen energy of a gluon in the lab frame is $E_{g}=\sqrt{k_{T}^{2}+\left(\frac{n \pi}{L}\right)^{2}}$, where $k_{T}$ is the transverse momentum of the gluon and $n=1,2 \cdots$ is the quantum number of $k_{z}$. The loss term in Eqn. (2) is replaced by

$$
\alpha_{H}=\frac{1}{2 E_{H} L} \sum_{n} \int \frac{\mathrm{d}^{2} \boldsymbol{k}_{T}}{(2 \pi)^{2} 2 E_{g}} W_{g H}^{Q \bar{Q}}(s) f_{g}\left(\boldsymbol{k}_{T}, n, \boldsymbol{x}, t\right)
$$

where $f_{g}\left(\boldsymbol{k}_{T}, n, \boldsymbol{x}, t\right)$ takes the same form as in Eqn. (4) with $k_{z}=n \pi / L$. Note that Eqn. (4) is invariant under a transverse boost. Eqn. (6) can be rewritten in the quarkonium frame as

$$
\alpha_{H}=\frac{1}{4 E_{H}} \int \frac{\mathrm{d} \omega}{\omega} f_{\omega} W_{g H}^{Q \bar{Q}}(s)
$$

The number density $f_{\omega}$ of gluons in unit energy is

$$
f_{\omega}=\frac{\mathrm{d} N}{\mathrm{~d} \omega \mathrm{d} V}=\frac{1}{L} \sum_{n} \int \frac{\mathrm{d}^{2} \boldsymbol{k}_{T}^{\prime}}{(2 \pi)^{2} \mathrm{~d} \omega} f_{g}\left(\boldsymbol{k}_{T}^{\prime}, n, \boldsymbol{x}, t\right),
$$

with the gluon thermal distribution function

$$
f_{g}\left(\boldsymbol{k}_{T}^{\prime}, n, \boldsymbol{x}, t\right)=\frac{N_{g}}{e^{u_{H}^{\mu} k_{\mu}^{\prime} / T}-1}
$$

where $u_{H}^{\mu}$ and $k_{\mu}^{\prime}$ are, respectively, the four-velocity and four-momentum in the quarkonium frame. 


\section{Modified Euler-Maclaurin Formula}

In order to work out the summation in Eqn. (8), we develop a modified Euler-Maclaurin formula. The original Euler-Maclaurin formula [38] is

$$
\sum_{i=a}^{b} f(i)=\int_{a}^{b} f(x) \mathrm{d} x+\frac{f(b)+f(a)}{2}+\sum_{r=1}^{\left[\frac{n}{2}\right]} \frac{B_{2 r}}{(2 r) !}\left(f^{(2 r-1)}(b)-f^{(2 r-1)}(a)\right)+R_{n}
$$

where $B_{2 r}$ is the $(2 \mathrm{r})$ th Bernoulli number [39]. The remainder term is

$$
R_{n}=\frac{(-1)^{n+1}}{n !} \int_{a}^{b} f^{(n)}(x) P_{n}(x) \mathrm{d} x
$$

where $P_{n}(x)$ is the periodic Bernoulli polynomial [40].

Sometimes the first a few terms are important (e.g. low energy states in calculating the partition function of bosons at low temperature). Thus we take the summation of the first $m$ terms explicitly. The Bernoulli number $B_{2 r}$ grows fast with $r$, and the remainder term $R_{n}$ often diverges as $n \rightarrow \infty$. The Fourier series of $P_{n}$ is [41]

$$
P_{n}(x)=-n ! \sum_{k \in Z-\{0\}} \frac{e^{2 \pi i k x}}{(2 \pi i k)^{n}}
$$

We take $2 p$ terms with $|k| \leqslant p$ in Eqn. (12) and leave the others to the new reminder term $R_{m n p}$. Then the modified Euler-Maclaurin formula is

$$
\begin{aligned}
\sum_{i=a}^{b} f(i)= & \sum_{i=a}^{a^{\prime}-1} f(i)+\frac{f(b)+f\left(a^{\prime}\right)}{2}+\int_{a^{\prime}}^{b} f(x) \frac{\sin (2 p+1) \pi x}{\sin \pi x} \mathrm{~d} x \\
& +\sum_{r=1}^{\left[\frac{n}{2}\right]} \frac{2(-1)^{r-1}}{(2 \pi)^{2 r}} S_{2 r, p} M_{2 r-1}\left(b, a^{\prime}\right)+R_{m n p}
\end{aligned}
$$

with $S_{2 r, p}=\zeta(2 r)-\sum_{k=1}^{p} \frac{1}{k^{2 r}}, M_{2 r-1}\left(b, a^{\prime}\right)=f^{(2 r-1)}(b)-f^{(2 r-1)}\left(a^{\prime}\right)$, and $a^{\prime}=a+m$. Here the $\zeta$ in $S_{2 r, p}$ is the Riemann zeta function. Dropping the new remainder term

$$
R_{m n p}=2 \sum_{k=p+1}^{\infty} \int_{a^{\prime}}^{b} f(x) \cos (2 \pi k x) \mathrm{d} x+\sum_{r=1}^{\left[\frac{n}{2}\right]} \frac{2(-1)^{r}}{(2 \pi)^{2 r}} S_{2 r, p} M_{2 r-1}\left(b, a^{\prime}\right),
$$

a m-n-p cut of the modified Euler-Maclaurin formula is obtained, which can be used as a numerical algorithm of the original summation. Any accuracy can be achieved by choosing $n$ and $p$. In practice, the integral in Eqn. (13) can also be calculated by

$$
\int_{a^{\prime}}^{b} f(x) \frac{\sin (2 p+1) \pi x}{\sin \pi x} \mathrm{~d} x=2 \sum_{k=1}^{p} \int_{a^{\prime}}^{b} f(x) \cos (2 \pi k x) \mathrm{d} x+\int_{a^{\prime}}^{b} f(x) \mathrm{d} x .
$$


The loss term in Eqn. (77) is calculated by a 2-2-1 cut of the modified Euler-Maclaurin formula as

$$
\alpha_{H}=\sum_{i=1}^{2} H(i)+\int_{3}^{\infty} H(y) \frac{\sin 3 \pi y}{\sin \pi y} \mathrm{~d} y+\frac{1}{2} H(3)-\frac{2}{(2 \pi)^{2}}(\zeta(2)-1) H^{(1)}(3)
$$

in the next section with

$$
H(y)=\frac{1}{4 E_{H} L} \int \frac{\mathrm{d}^{2} \boldsymbol{k}_{T}^{\prime}}{(2 \pi)^{2} \omega} W_{g H}^{Q \bar{Q}}(s) f_{g}\left(\boldsymbol{k}_{T}^{\prime}, y, \boldsymbol{x}, t\right) .
$$

\section{Numerical Results}

Now we discuss the number density $f_{\omega}$ in unit energy in Eqn. (8) of gluons which is called density in the following for short. Fig. 1 1 shows the density as a function of $\omega$ in a

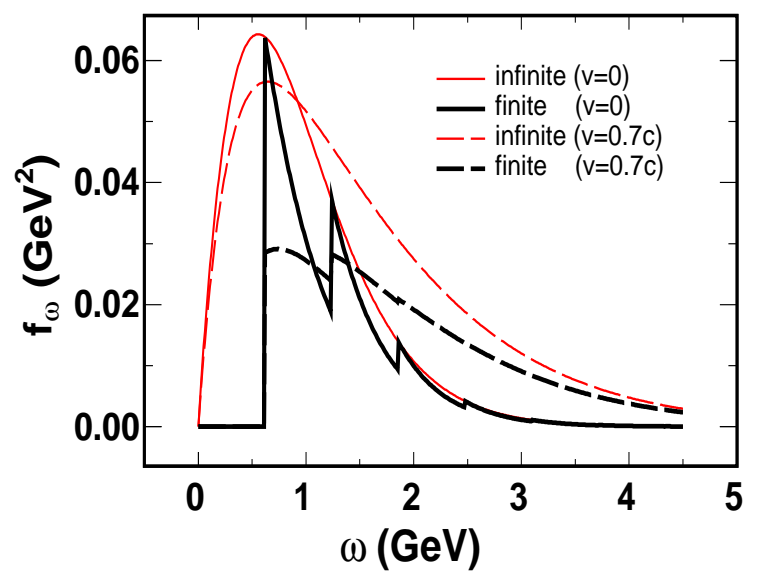

Figure 1: (Color online) The gluon number density $f_{\omega}$ in unit energy in the finite space $(L=1 \mathrm{fm})$ and the infinite space $(L=\infty)$ at $T=0.35 \mathrm{GeV}$ with $v=0$ and $v=0.7 c$.

static $(v=0)$ or moving frame $(v=0.7 c)$ in a finite fireball $(L=1 \mathrm{fm})$ compared with that in an infinite fireball $(L=\infty)$ at $T=0.35 \mathrm{GeV}$. The density in a finite fireball is never larger than that in an infinite fireball and the gluons whose energy is less than the ground state energy $\omega_{0}=\pi / L$ vanish. In order to understand the properties of $f_{\omega}$, we consider two limits: in the static frame and in the fast moving one. (i) In the static frame $(v=0)$, Eqn. (8) can be simplified as

$$
f_{\omega}^{L}=\frac{N_{g}}{2 \pi L}\left[\frac{\omega}{\omega_{0}}\right] \frac{\omega}{e^{\omega / T}-1}
$$

while the density in the infinite space is

$$
f_{\omega}^{\infty}=\frac{N_{g}}{2 \pi^{2}} \frac{\omega^{2}}{e_{5}^{\omega / T}-1} .
$$


The ratio $f_{r}=f_{\omega}^{L} / f_{\omega}^{\infty}$ satisfies $\left(1-\frac{\omega_{0}}{\omega}\right) \leqslant f_{r} \leqslant 1$ at $\omega \geqslant \omega_{0}$ and the equality holds only at $\omega=n \omega_{0}(n=1,2,3 \cdots)$ as shown in Fig. 11 (ii) In the fast moving frame, Eqn. (8) can be simplified in the condition of both $\left|u_{H}^{T}\right| \gg 2 T \omega / \omega_{0}^{2}$ and $\left|u_{H}^{T}\right| \gg T / \omega$ as

$$
f_{\omega}^{L}=\frac{N_{g}}{L} \sqrt{\frac{\omega}{(2 \pi)^{3} B}} e^{-A \omega+B \omega \sqrt{1-\left(\frac{\omega_{0}}{\omega}\right)^{2}}},
$$

with $A=u_{H}^{0} / T$ and $B=\left|u_{H}^{T}\right| / T$. In the infinite space, the density is

$$
f_{\omega}^{\infty}=\frac{N_{g} \omega}{(2 \pi)^{2} B} e^{(-A+B) \omega} .
$$

The ratio in the fast moving frame is

$$
f_{r}=\frac{1}{L} \sqrt{\frac{2 \pi B}{\omega}} e^{B \omega\left(\sqrt{1-\left(\frac{\omega_{0}}{\omega}\right)^{2}}-1\right)} \ll 1
$$

which shows strong suppression of the density in the finite space.

The change of the density leads to the change of the loss term $\alpha_{H}$ defined in Eqn. (7). As a result of the discussion in the previous paragraph, in the static frame, the loss

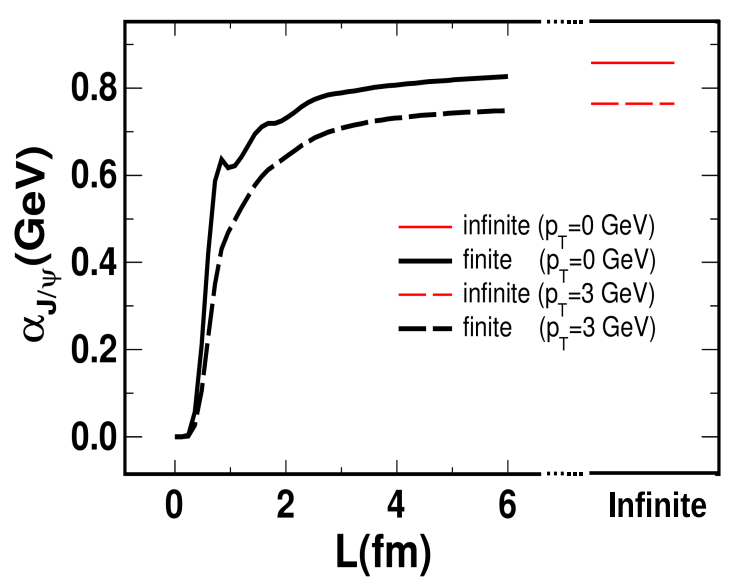

Figure 2: (Color online) The loss term $\alpha_{J / \psi}$ as a function of longitudinal size $L$ at transverse momentum $p_{T}=0$ and $3 \mathrm{GeV}$ at $T=0.35 \mathrm{GeV}$.

term $\alpha^{L}$ in the finite space lies between $\frac{1}{2} \alpha^{\infty}$ and $\alpha^{\infty}$ at $\omega_{0}<\epsilon_{H}$, and it is far smaller than $\alpha^{\infty}$ at $\omega_{0} \gg \epsilon_{H}$ with $\omega_{0} \gtrsim T$. In the following we discuss the dissociation rate of $J / \psi$ in two cases. (i) We fix the temperature $T=0.35 \mathrm{GeV}$ as a constant. Fig. 2 shows the loss term $\alpha_{J / \psi}$ as a function of $L$ at the transverse momentum $p_{T}=0$ and 3 $\mathrm{GeV}$ of $J / \psi$. The finite system approaches to the infinite system when $L$ is large, and the finite volume effect is remarkable at a small $L\left(\lesssim \frac{\pi}{\epsilon_{J / \psi}}\right)$. The kinks of the line in Fig. 2 come from the jumps of $f_{\omega}$ in Fig. 1. (ii) We evolve temperature $T$ according to the Bjorken's hydrodynamics. In Fig. 3, we show the $\alpha_{J / \psi}$ as a function of time $t$ with transverse momentum $p_{T}=0$ and $3 \mathrm{GeV}$ with $T^{*}=0.35 \mathrm{GeV}$ at $t^{*}=0.6 \mathrm{fm} / c$. In 


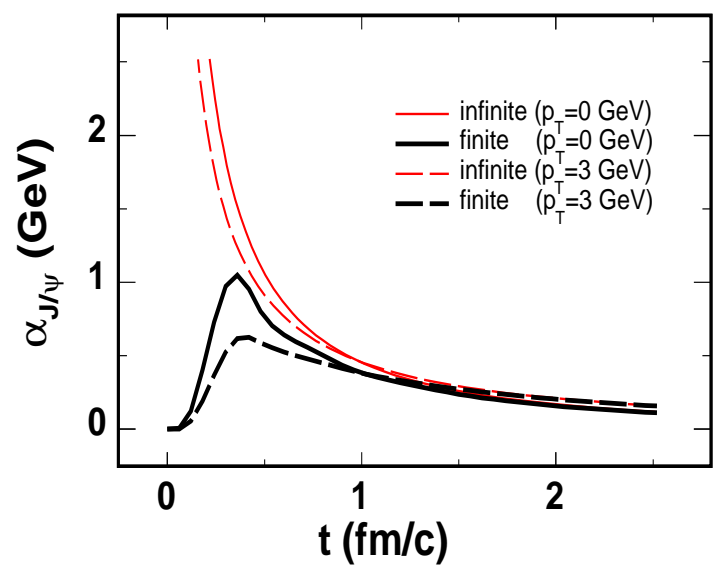

Figure 3: (Color online) The loss term $\alpha_{J / \psi}$ as a function of the time $t$ in the finite space and the infinite space with the transverse momentum $p_{T}=0$ and $3 \mathrm{GeV}$ of $J / \psi$ with Bjorken's hydrodynamics.

the infinite space, the loss term is divergent at $t=0$. This divergence is usually avoided by constraining the suppression after the formation of the QGP and $J / \psi$. However our calculation indicates that, even if the formation times of the QGP and $J / \psi$ are early enough, the loss term $\alpha_{J / \psi}$ is still negligible at small $t$. The results of $\Upsilon(1 s)$ are similar.

We define the effective initial time $t_{0}$ by requiring the suppression in the finite space since $t=0$ is equal to that in the infinite space since $t=t_{0}$ which should be an additional cut besides the ordinary two formation times as mentioned above. The calculated effective initial time $t_{0}$ of $H(J / \psi, \Upsilon(1 s))$ as a function of $p_{T}$ with temperature $T^{*}=0.35$ and $0.51 \mathrm{GeV}$ at $t^{*}=0.6 \mathrm{fm} / c$ is shown in Fig. 4. The threshold energy $\epsilon_{H}$ is important

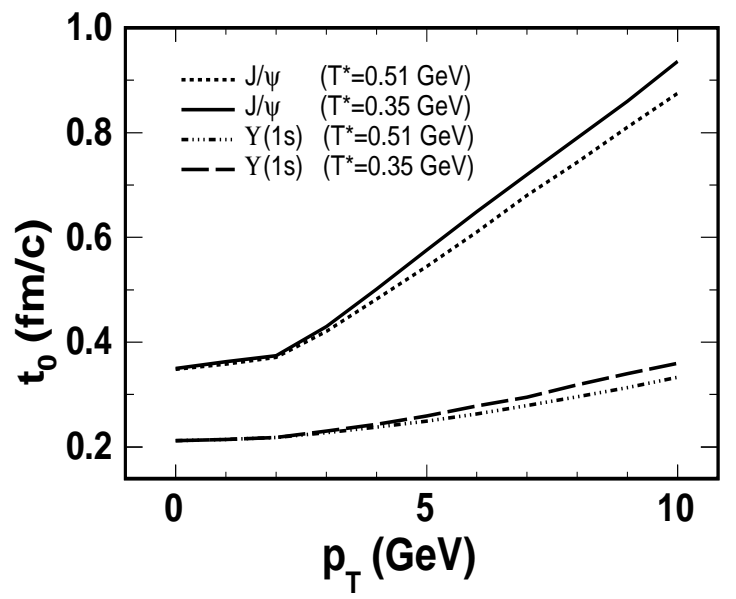

Figure 4: The effective initial time $t_{0}$ of $J / \psi$ and $\Upsilon(1 s)$ as a function of $p_{T}$ with Bjorken's hydrodynamics with $T^{*}=0.35$ and $0.51 \mathrm{GeV}$, respectively, at $t^{*}=0.6 \mathrm{fm} / c$. 
to the effective initial time. As discussed in previous paragraph, in the static frame, we have $\alpha^{L} \sim \alpha^{\infty}$ at $\omega_{0} \ll \epsilon_{H}$, and $\alpha^{L} \ll \alpha^{\infty}$ at $\omega_{0} \gg \epsilon_{H}$ with $\omega_{0} \gtrsim T$. Therefore, $\omega_{0}=\epsilon_{H}$ gives a rough estimate of $t_{0}$, which leads to the relation $t_{0_{J / \psi}} / t_{0_{\Upsilon(1 s)}} \approx \epsilon_{\Upsilon(1 s)} / \epsilon_{J / \psi}$. In our calculation, the ratio of $\epsilon_{\Upsilon(1 s)} / \epsilon_{J / \psi}$ and $t_{0_{J / \psi}} / t_{0_{\Upsilon(1 s)}}$ are 1.67 and 1.65 , respectively. At high- $p_{T}$, the suppression of $f_{\omega}$ is strong according to Eqn. (22). Therefore $t_{0}$ increases monotonically with $p_{T}$. No strong dependence of $t_{0}$ on the initial local temperature $T^{*}$ is observed in our calculation.

\section{Conclusion}

Based on the rate equation of heavy quarkonia and the Bjorken's hydrodynamics, we calculated the gluon number density $f_{\omega}$ in unit energy and the loss term $\alpha_{J / \psi}$ in the finite space compared with that in the infinite space at the same temperature. It is found that the suppression of heavy quarkonia in the constrained space is weak, and therefore the suppression of heavy quarkonia at early time after the collision can be neglected, even if the temperature of the medium is high. The resulting concept effective initial time $t_{0}$ can be estimated at $p_{T}=0$ by the threshold energy, and it increases with $p_{T}$. A modified Euler-Maclaurin formula is developed to deal with the summation powerfully.

\section{Acknowledgments}

The work is supported by the NSFC under the Grant No.s 11547043, 11705125 and by the "Qinggu" project of Tianjin University.

\section{References}

\section{References}

[1] M. C. Abreu, et al., Observation of a threshold effect in the anomalous J/psi suppression, Phys. Lett. B450 (1999) 456-466. doi:10.1016/S0370-2693(99) 00178-1

[2] A. Adare, et al., $J / \psi$ production vs centrality, transverse momentum, and rapidity in $\mathrm{Au}+\mathrm{Au}$ collisions at $\sqrt{s_{N N}}=200 \mathrm{GeV}$, Phys. Rev. Lett. 98 (2007) 232301. arXiv:nucl-ex/0611020 doi:10.1103/PhysRevLett.98.232301

[3] L. Adamczyk, et al., $J / \psi$ production at high transverse momenta in $p+p$ and $\mathrm{Au}+\mathrm{Au}$ collisions at $\sqrt{s_{N N}}=200 \mathrm{GeV}$, Phys. Lett. B722 (2013) 55-62. arXiv:1208.2736 doi:10.1016/j.physletb.2013.04.010

[4] S. Chatrchyan, et al., Suppression of non-prompt $J / \psi$, prompt $J / \psi$, and $\mathrm{Y}(1 \mathrm{~S})$ in $\mathrm{Pb}-\mathrm{Pb}$ collisions at $\sqrt{s_{N N}}=2.76 \mathrm{TeV}$, JHEP 05 (2012) 063. arXiv:1201.5069, doi:10.1007/JHEP05(2012) 063

[5] B. Abelev, et al., $J / \psi$ suppression at forward rapidity in $\mathrm{Pb}-\mathrm{Pb}$ collisions at $\sqrt{s_{N N}}=2.76 \mathrm{TeV}$, Phys. Rev. Lett. 109 (2012) 072301. arXiv:1202.1383 doi:10.1103/PhysRevLett.109.072301

[6] A. Adare, et al., $J / \psi$ suppression at forward rapidity in $\mathrm{Au}+\mathrm{Au}$ collisions at $\sqrt{s_{N N}}=200 \mathrm{GeV}$, Phys. Rev. C84 (2011) 054912. arXiv:1103.6269 doi:10.1103/PhysRevC.84.054912

[7] T. Matsui, H. Satz, $J / \psi$ suppression by quark-gluon plasma formation, Phys. Lett. B178 (1986) 416-422. doi:10.1016/0370-2693(86) 91404-8

[8] J. Blaizot, J. Ollitrault, $J / \psi$ suppression in $\mathrm{Pb}-\mathrm{Pb}$ collisions: A hint of quark-gluon plasma production?, Phys. Rev. Lett. 77 (1996) 1703-1706. arXiv:hep-ph/9606289 doi:10.1103/PhysRevLett.77.1703

[9] P. BraunMunzinger, J. Stachel, (Non)thermal aspects of charmonium production and a new look at J/psi suppression, Phys. Lett. B490 (2000) 196-202. arXiv:nucl-th/0007059 doi:10.1016/S0370-2693(00)00991-6

[10] X. Xu, D. Kharzeev, H. Satz, X. Wang, J/psi suppression in an equilibrating parton plasma, Phys. Rev. C53 (1996) 3051-3056. arXiv:hep-ph/9511331, doi:10.1103/PhysRevC.53.3051 
[11] Y. Oh, T. Song, S. H. Lee, J/psi absorption by pi and rho mesons in meson exchange model with anomalous parity interactions, Phys. Rev. C63 (2001) 034901. arXiv:nucl-th/0010064. doi:10.1103/PhysRevC.63.034901

[12] L. Grandchamp, R. Rapp, Thermal versus direct J/Psi production in ultrarelativistic heavy ion collisions, Phys. Lett. B523 (2001) 60-66. arXiv:hep-ph/0103124 doi:10.1016/S0370-2693(01)01311-9

[13] P. Zhuang, X. Zhu, Leakage effect on $\mathrm{J} / \mathrm{psi} \mathrm{p}(\mathrm{t})$ distributions in different centrality bins for $\mathrm{Pb} \mathrm{Pb}$ collisions at $\mathrm{E} / \mathrm{A}=160 \mathrm{GeV}$, Phys. Rev. C67 (2003) 067901. arXiv:nucl-th/0304019 doi:10.1103/PhysRevC.67.067901

[14] R. L. Thews, M. Schroedter, J. Rafelski, Enhanced $J / \psi$ production in deconfined quark matter, Phys. Rev. C63 (2001) 054905. arXiv:hep-ph/0007323 doi:10.1103/PhysRevC.63.054905

[15] L. Yan, P. Zhuang, N. Xu, Competition between J/psi suppression and regeneration in quark-gluon plasma, Phys. Rev. Lett. 97 (2006) 232301. arXiv:nucl-th/0608010 doi:10.1103/PhysRevLett.97.232301

[16] N. Brambilla, A. Pineda, J. Soto, A. Vairo, Potential NRQCD: an effective theory for heavy quarkonium, Nucl. Phys. B566 (2000) 275. arXiv:hep-ph/9907240, doi:10.1016/S0550-3213(99) 00693-8

[17] M. Asakawa, T. Hatsuda, J/psi and eta(c) in the deconfined plasma from lattice QCD, Phys. Rev. Lett. 92 (2004) 012001. arXiv:hep-lat/0308034, doi:10.1103/PhysRevLett.92.012001

[18] X. Zhao, R. Rapp, Transverse Momentum Spectra of $J / \psi$ in Heavy-Ion Collisions, Phys. Lett. B664 (2008) 253-257. arXiv:0712.2407 doi:10.1016/j.physletb.2008.03.068

[19] F. Riek, R. Rapp, Quarkonia and heavy-quark relaxation times in the quark-gluon plasma, Phys. Rev. C82 (2010) 035201. arXiv:1005.0769 doi:10.1103/PhysRevC.82.035201

[20] J. Uphoff, O. Fochler, Z. Xu, C. Greiner, Heavy quark production at RHIC and LHC within a partonic transport model, Phys. Rev. C82 (2010) 044906. arXiv:1003.4200 doi:10.1103/PhysRevC.82.044906

[21] H. R. Grigoryan, P. M. Hohler, M. A. Stephanov, Towards the Gravity Dual of Quarkonium in the Strongly Coupled QCD Plasma, Phys. Rev. D82 (2010) 026005. arXiv:1003.1138 doi:10.1103/PhysRevD.82.026005

[22] T. Song, K. C. Han, C. M. Ko, The effect of initial fluctuations on bottomonia suppression in relativistic heavy-ion collisions arXiv:1112.0613

[23] Y. Liu, N. Xu, P. Zhuang, Velocity dependence of charmonium dissociation temperature in high-energy nuclear collisions, Phys. Lett. B724 (2013) 73-76. arXiv:1210.7449 doi:10.1016/j.physletb.2013.05.068

[24] Y. Liu, C. M. Ko, T. Song, Gluon dissociation of J/psi beyond the dipole approximation, Phys. Rev. C88 (6) (2013) 064902. arXiv:1307.4427 doi:10.1103/PhysRevC.88.064902

[25] T. Song, C. M. Ko, S. H. Lee, Quarkonium formation time in quark-gluon plasma, Phys. Rev. C87 (3) (2013) 034910. arXiv:1302.4395 doi:10.1103/PhysRevC.87.034910

[26] K. Zhou, N. Xu, Z. Xu, P. Zhuang, Medium effects on charmonium production at ultrarelativistic energies available at the CERN Large Hadron Collider, Phys. Rev. C89 (5) (2014) 054911 arXiv:1401.5845 doi:10.1103/PhysRevC.89.054911

[27] H. Ding, F. Karsch, S. Mukherjee, Thermodynamics of strong-interaction matter from Lattice QCD, Int. J. Mod. Phys. E24 (10) (2015) 1530007. arXiv:1504.05274 doi:10.1142/S0218301315300076

[28] B. Chen, X. Du, R. Rapp, Internal charmonium evolution in the quarkgluon plasma, Nucl. Part. Phys. Proc. 289-290 (2017) 475-478. arXiv:1612.02089 doi:10.1016/j.nuclphysbps.2017.05.111.

[29] W. Shi, W. Zha, B. Chen, Charmonium coherent photoproduction and hadroproduction with effects of quark gluon plasma, Phys. Lett. B777 (2018) 399-405. arXiv:1710.00332 doi:10.1016/j.physletb.2017.12.055

[30] S. Chen, M. He, Heavy quarkonium dissociation by thermal gluons at next-to-leading order in the quarkgluon plasma, Phys. Lett. B786 (2018) 260-267. arXiv:1805.06346 doi:10.1016/j.physletb.2018.09.056

[31] X. Zhu, P. Zhuang, N. Xu, J/psi transport in QGP and p(t) distribution at SPS and RHIC, Phys. Lett. B607 (2005) 107-114. arXiv:nucl-th/0411093 doi:10.1016/j.physletb.2004.12.023

[32] B. Chen, K. Zhou, P. Zhuang, Mean Field Effect on $J / \psi$ Production in Heavy Ion Collisions, Phys. Rev. C86 (2012) 034906. arXiv:1202.3523 doi:10.1103/PhysRevC.86.034906

[33] M. E. Peskin, Short distance analysis for heavy quark systems. 1. diagrammatics, Nucl. Phys. B156 (1979) 365-390. doi:10.1016/0550-3213(79)90199-8

[34] G. Bhanot, M. E. Peskin, Short distance analysis for heavy quark systems. 2. applications, Nucl. Phys. B156 (1979) 391-416. doi:10.1016/0550-3213(79)90200-1 
[35] Y. Liu, Z. Qu, N. Xu, P. Zhuang, J/psi Transverse Momentum Distribution in High Energy Nuclear Collisions at RHIC, Phys. Lett. B678 (2009) 72-76. arXiv:0901.2757 doi:10.1016/j.physletb.2009.06.006

[36] M. L. Miller, K. Reygers, S. J. Sanders, P. Steinberg, Glauber modeling in high energy nuclear collisions, Ann. Rev. Nucl. Part. Sci. 57 (2007) 205-243. arXiv:nucl-ex/0701025 doi:10.1146/annurev.nucl.57.090506.123020

[37] C. W. De Jager, H. De Vries, C. De Vries, Nuclear charge and magnetization density distribution parameters from elastic electron scattering, Atom. Data Nucl. Data Tabl. 14 (1974) 479-508. doi:10.1016/S0092-640X (74)80002-1

[38] T. M. Apostol, An elementary view of euler's summation formula The American Mathematical Monthly 106 (5) (1999) 409-418. URL http://www.jstor.org/stable/2589145

[39] M. Abramowitz, I. A. Stegun, Handbook of mathematical functions with formulas, graphs, and mathematical Tables, Dover Publications, 1972.

[40] D. H. Lehmer, On the maxima and minima of Bernoulli Polynomials, The American Mathematical Monthly 47 (8) (1940) 533-538. arXiv:https://doi.org/10.1080/00029890.1940.11991015 doi:10.1080/00029890.1940.11991015

[41] Q. M. Luo, Fourier expansions and integral representations for the Apostol-Bernoulli and Apostol-Euler polynomials, Mathematics of Computation 78 (268) (2010) 2193-2208. doi:https://doi.org/10.1090/S0025-5718-09-02230-3 